\title{
Polycystic ovarian morphology is associated with primary dysmenorrhea in young Korean women
}

\author{
Jee Young Jeong ${ }^{1,2}$, Min Kyoung Kim ${ }^{4}$, Inha Lee ${ }^{1,2}$, Jisun Yun ${ }^{1,2}$, Young Bin Won ${ }^{1,2}$, Bo Hyon Yun ${ }^{1,2}$, \\ Seok Kyo Seo ${ }^{1,2}$, SiHyun Cho ${ }^{2,3}$, Young Sik Choi ${ }^{1,2}$, Byung Seok Lee ${ }^{1,2}$ \\ ${ }^{1}$ Department of Obstetrics and Gynecology, Severance Hospital, ${ }^{2}$ Institute of Women's Life Medical Science, ${ }^{3}$ Department of Obstetrics and \\ Gynecology, Gangnam Severance Hospital, Yonsei University College of Medicine; ${ }^{4}$ Fertility Center of CHA Gangnam Medical Center, CHA University \\ School of Medicine, Seoul, Korea
}

\section{Objective}

This study was aimed at identifying a correlation between polycystic ovarian morphology (PCOM) and the severity of primary dysmenorrhea in young Korean women.

\section{Methods}

A total of 592 patients who visited a tertiary hospital from March 2008 to March 2015 for dysmenorrhea were examined. After excluding those with secondary causes of menstrual pain (for example, myoma, adenomyosis, endometriosis, and pelvic inflammatory disease), 361 women were recruited and retrospectively analyzed. Severe dysmenorrhea was defined as a visual analog scale (VAS) score $\geq 6$.

\section{Results}

The mean patient age was $23.0 \pm 4.0$ years, the average menstrual cycle length was $34.4 \pm 23.7$ days, and the average pain intensity was VAS $6.7 \pm 0.1$ at baseline. PCOM was assessed by ultrasound in 54 women (15\%). Patients with severe menstrual pain were more likely to have irregular menstrual cycles $(P=0.03)$ and heavy menstrual flow $(P=0.01)$ than those with mild menstrual pain. After adjusting for weight, height, menstrual cycle interval, and menstrual flow in the logistic regression analysis, PCOM (odds ratio [OR], 2.26; 95\% confidence interval $[\mathrm{Cl}], 1.05-4.97 ; P=0.04$ ) and heavy menstrual flow $(\mathrm{OR}, 1.85 ; 95 \% \mathrm{Cl}, 1.05-3.28 ; P=0.04)$ were found to be significant independent factors influencing pain.

\section{Conclusion}

Our study shows that PCOM may have a correlation with the severity of primary dysmenorrhea. Since PCOM may play a role in the development of menstrual pain, patients with PCOM should be under active surveillance with resources for prompt pain management readily available. It may also be necessary to further investigate the molecular mechanisms of pain development in primary dysmenorrhea.

Keywords: Dysmenorrhea; Menstruation disturbances; Polycystic ovary syndrome

\section{Introduction}

Primary dysmenorrhea is defined as menstrual pain associated with ovulatory cycles and resulting from myometrial contraction in the absence of a pelvic disease that may cause abdominal pain. Traditionally, the activity of prostaglandin, a vasoconstrictor that causes ischemic pain in the uterus, is regarded as the cause of primary dysmenorrhea [1]. Although primary dysmenorrhea is common, it is frequently underdiagnosed and under-treated [2]. Identification of the risk factors of primary dysmenorrhea may be useful for classifying
Received: 2018.08.26. Revised: 2018.11.03. Accepted: 2018.11.08. Corresponding author: Bo Hyon Yun

Department of Obstetrics and Gynecology, Severance Hospital, Yonsei University College of Medicine, 50 Yonsei-ro, Seodaemungu, Seoul 03722, Korea

E-mail: garfieldzz@yuhs.ac

https://orcid.org/0000-0001-5703-797X

Articles published in Obstet Gynecol Sci are open-access, distributed under the terms of the Creative Commons Attribution Non-Commercial License (http://creativecommons. org/licenses/by-nc/3.0/) which permits unrestricted non-commercial use, distribution, and reproduction in any medium, provided the original work is properly cited.

Copyright $\odot 2019$ Korean Society of Obstetrics and Gynecology 


\title{
Obstetrics \& Gynecology Science
}

\author{
Vol. 62, No. 5, 2019
}

women into groups that need active surveillance or prompt pain management.

Polycystic ovarian morphology (PCOM), one of the diagnostic criteria for polycystic ovary syndrome (PCOS), is defined as the presence of $>12$ antral follicles $2-9 \mathrm{~mm}$ in diameter or an ovarian volume $>10 \mathrm{~mL}$ [3]. The inclusion of PCOM in the diagnostic criteria for PCOS is controversial because the same morphology is found in 16-25\% of asymptomatic women [4]. In a small study, the prevalence of PCOM was reported to be $35-50 \%$ in healthy adolescent girls who were neither diagnosed with nor treated for PCOS 2-4 years after menarche [5-7]. The inclusion of PCOM to the PCOS spectrum is a topic of debate because ovulatory women with isolated PCOM frequently do not show clinical or biochemical evidence of androgen excess [8]. The clinical significance of PCOM is unclear. To date, PCOM is considered a pre-PCOS state, a normal morphological variant in adolescence, and a possible complication in high responders to controlled ovarian hyperstimulation [9].

The purpose of our study was to identify factors that may have a correlation with the severity of primary dysmenorrhea, and to investigate the relationship between PCOM and primary dysmenorrhea.

\section{Materials and methods}

\section{Study population}

A comparative study was conducted using a retrospective chart review of a total of 592 young, postmenarcheal Korean women aged $<30$ years old, who visited Severance Hospital, Yonsei University College of Medicine, between March 2008 and March 2015 with dysmenorrhea as their chief complaint. After initial evaluation, 231 women were excluded for other possible causes of dysmenorrhea (Fig. 1). The initial evaluation included history taking, pelvic examination, and imaging studies with either pelvic sonography (Accuvix V20 Prestige, Medison Co., Seoul, Korea) or abdominopelvic computed tomography. On their initial visit and using questionnaires, the patients provided information on their menstrual history which included cycle regularity and duration, quantity of menstruation, pain onset, any predictable patterns such as pain development timing and duration, any accompanying symptoms such as back pain, vomiting, diarrhea, or intermenstrual bleeding. Patients diagnosed with pelvic masses such as myoma and adnexal cyst, endometrial pathologies like polyp or submucosal myoma, endometriosis, and adenomyosis on imaging and patients with suspected symptoms of pelvic pathology were excluded from the analysis. Patients who, in the 3 months prior to their hospital visit, had taken hormonal agents such as oral contraceptives or progestin were also excluded. Finally, a total of 361 women with primary dysmenorrhea were recruited for the study [10].

\section{Variables and measurements}

Baseline physical examinations included measurements of height $(\mathrm{cm})$, weight $(\mathrm{kg})$, and body mass index $\left(\mathrm{BMl}, \mathrm{kg} / \mathrm{m}^{2}\right)$. The pain intensity of dysmenorrhea was quantified using a visual analog scale (VAS) with scores from 0 to 10 . Severe dysmenorrhea was defined as a VAS score $\geq 6$. Irregular menstruation was defined in traditional terms as a cycle interval $>35$ days or $<21$ days. Menstrual flow was classified as scanty, moderate, or heavy and PCOM was defined according to the Rotterdam criteria [3]. Sonography was performed according to menstrual cycle; in the early follicular phase for patients with regular periods or with a dominant follicle or corpus luteum on ultrasound scan, and regardless of the ovulation cycle for patients with irregular periods of over 90 days or amenorrhea. In patients with sonographic evidence of ovarian cyst, the criteria for PCOM was applied using the 2-9 $\mathrm{mm}$ antral follicle count only. Smoking status was regarded as positive for current smokers only. The medications administered for menstrual pain relief after consulta-

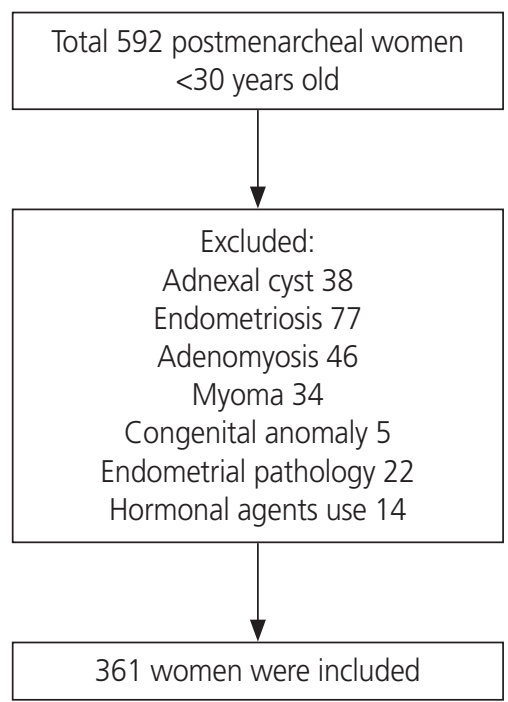

Fig. 1. Flow chart of the participant inclusion in the study. 


\section{Obstetrics \& Gynecology Science}

Jee Young Jeong, et al. PCOM and primary dysmenorrhea

tion were classified as none (no medication use), nonsteroidal anti-inflammatory drugs, and combined oral contraceptives, and information on the use of pain relief medications of different groups was considered.

\section{Statistical analyses}

Data are presented as mean \pm standard deviation for continuous variables and as numbers and percentages for categorical variables. The baseline characteristics between the mild and severe dysmenorrhea groups were compared using a 2 -sample $t$-test for continuous variables and the $\chi^{2}$ test for categorical variables. Logistic regression analyses were performed to identify factors that are associated with severe primary dysmenorrhea. Logistic models were built based on the model's goodness-of-fit and the multicollinearity of the factors. Previously known factors for primary dysmenorrhea with a $P$-value $<0.1$ in the univariate analysis were included in the logistic model. Data were analyzed using IBM SPSS ver. 23.0 (IBM Corp., Armonk, NY, USA). A P-value $<0.05$ was considered statistically significant.

\section{Results}

A total of 361 patients with primary dysmenorrhea were included in the analyses. Their baseline characteristics are shown in Table 1. The mean age of the patients was $23.0 \pm 4.0$ years, the mean menstrual cycle duration was $34.4 \pm 23.7$ days, and the mean pain intensity measured on the VAS scale was $6.7 \pm 0.1$. The mean BMI was $20.7 \pm 3.0 \mathrm{~kg} / \mathrm{cm}^{2}$, which was within the normal range. Of the 361 patients, $155(42.9 \%)$ reported having mild dysmenorrhea (VAS $\leq 5$ ) and 206 (57.1\%) reported having severe dysmenorrhea (VAS 26). Pelvic ultrasound examination of a total of 54 women (15\%) showed PCOM. Baseline characteristics such as BMI and menstrual cycle duration were similar between women with mild dysmenorrhea and those with severe dysmenorrhea (Table 2). However, the proportion of women who had irregular menstrual cycles was significantly higher in the severe dysmenorrhea group ( $P=0.03)$. Furthermore, women with severe pain reported heavier menstrual flow than those with mild pain $(P=0.02)$. The proportion of women with PCOM was also significantly higher in the severe dysmenorrhea group than in the mild dysmenorrhea group $(P=0.03)$. A statistically significant difference in medication use for pain relief was observed between the 2 groups, with a higher frequency of combined oral contraceptive use in the severe dysmenorrhea group than in the mild dysmenorrhea group ( $P=0.04$ ). Table 3 shows the adjustment model for women with severe primary dysmenorrhea. After adjusting for weight, height, menstrual cycle duration, and menstrual flow, PCOM showed a significantly increased odds ratio (OR) for severe dysmenorrhea (VAS score $\geq 6$; OR, 2.26; 95\% confidence interval $[\mathrm{Cl}], 1.05-4.97 ; P=0.04)$. Heavy menstrual flow was also associated with severe dysmenorrhea (OR, 1.85; $95 \% \mathrm{Cl}, 1.05-3.28 ; P=0.04)$.

\section{Discussion}

In this study, we investigated the factors associated with severe pain in patients with primary dysmenorrhea. Of fore-

Table 1. Baseline characteristics of patients with primary dysmenorrhea

\begin{tabular}{lc}
\hline Variables & Values \\
\hline Age $(\mathrm{yr})$ & $23.0 \pm 4.0$ \\
Weight $(\mathrm{kg})$ & $54.1 \pm 8.4$ \\
Height $(\mathrm{cm})$ & $161.7 \pm 5.3$ \\
Body mass index $\left(\mathrm{kg} / \mathrm{cm}^{2}\right)$ & $20.7 \pm 3.0$ \\
Menstrual cycle duration (day) & $34.4 \pm 23.7$ \\
Pain intensity (VAS) & $6.7 \pm 0.1$ \\
Menstrual flow & \\
Scanty & $24(6.6)$ \\
Moderate & $232(64.3)$ \\
Heavy & $86(23.8)$ \\
Dysmenorrhea & \\
Mild $($ VAS $\leq 5)$ & $155(42.9)$ \\
Severe $($ VAS $\geq 6)$ & $206(57.1)$ \\
PCOM & $54(15)$ \\
Current smoker & $9(3.2)$ \\
Pain medication & \\
None & $125(34.6)$ \\
NSAID & $102(28.3)$ \\
COC & $134(37.1)$ \\
\hline Da are &
\end{tabular}

Data are presented as mean \pm standard deviation for continuous variables and as number (\%) for categorical variables.

SD, standard deviation; VAS, visual analog scale; PCOM, polycystic ovarian morphology; NSAID, nonsteroidal anti-inflammatory drug; COC, combined oral contraceptive. 


\title{
Obstetrics \& Gynecology Science
}

\author{
Vol. 62, No. 5, 2019
}

Table 2. Comparison of the mild and severe dysmenorrhea groups

\begin{tabular}{|c|c|c|c|}
\hline Variables & Mild $(n=155)$ & Severe $(n=206)$ & $P$-value \\
\hline Age (yr) & $23.1 \pm 4.1$ & $22.9 \pm 4.0$ & 0.55 \\
\hline Weight (kg) & $53 \pm 7.8$ & $54.9 \pm 8.8$ & 0.05 \\
\hline Height (cm) & $160.8 \pm 5.2$ & $162.4 \pm 5.3$ & 0.01 \\
\hline Body mass index $\left(\mathrm{kg} / \mathrm{cm}^{2}\right)$ & $20.5 \pm 3.0$ & $20.8 \pm 3.0$ & 0.43 \\
\hline Menstrual cycle duration (day) & $33.7 \pm 28.7$ & $35.0 \pm 18.7$ & 0.61 \\
\hline Irregular period & $42(27.5)$ & $76(38.8)$ & 0.03 \\
\hline Menstrual flow & & & 0.02 \\
\hline Scanty+moderate & $121(81.2)$ & $135(69.9)$ & \\
\hline Heavy & $28(18.8)$ & $58(30.1)$ & \\
\hline PCOM & $16(10.3)$ & $38(18.4)$ & 0.03 \\
\hline Current smoker & $6(4.8)$ & $3(2)$ & 0.18 \\
\hline Pain medication & & & 0.04 \\
\hline None & $61(39.4)$ & $64(31.1)$ & \\
\hline NSAID & $48(31)$ & $54(26.2)$ & \\
\hline $\mathrm{COC}$ & $46(29.7)$ & $88(42.7)$ & \\
\hline
\end{tabular}

Data are presented as mean \pm standard deviation for continuous variables and as number (\%) for categorical variables. $P$-values were obtained using analysis of variance and 2-sample $t$-test for continuous variables, and the chi-square test for categorical variables.

PCOM, polycystic ovarian morphology; NSAID, nonsteroidal anti-inflammatory drug; COC, combined oral contraceptive.

Table 3. Adjusted odds ratios of variables for severe primary dysmenorrhea

\begin{tabular}{|c|c|c|c|c|}
\hline Variables & Unadjusted OR $(95 \% \mathrm{Cl})$ & $P$-value & Adjusted OR $(95 \% \mathrm{Cl})$ & $P$-value \\
\hline Weight & $1.03(1.0-1.06)$ & 0.05 & $1.02(0.98-1.05)$ & 0.38 \\
\hline Height & $1.06(1.01-1.11)$ & 0.01 & $1.04(0.99-1.10)$ & 0.11 \\
\hline \multicolumn{5}{|l|}{ Menstrual flow } \\
\hline Scanty+moderate & 1 & & 1 & \\
\hline Heavy & $1.86(1.11-3.1)$ & 0.02 & $1.85(1.05-3.28)$ & 0.04 \\
\hline PCOM & $1.97(1.05-3.67)$ & 0.03 & $2.26(1.05-4.97)$ & 0.04 \\
\hline Menstrual cycle duration & $1.0(0.99-1.01)$ & 0.62 & $1.0(0.99-1.01)$ & 0.72 \\
\hline
\end{tabular}

$P$-values were obtained using simple and multiple logistic regression analyses. The analyses were adjusted for weight (kg), height (cm), menstrual cycle duration (days), menstrual flow (reference: scanty+moderate), and PCOM (reference: no PCOM).

$\mathrm{OR}$, odds ratio; $\mathrm{Cl}$, confidence interval; PCOM, polycystic ovarian morphology.

most importance was our aim to ascertain the clinical significance of PCOM beyond its potential status as a pre-PCOS state. The results revealed that PCOM and heavy menstrual flow may be associated with severe primary dysmenorrhea in young Korean women.

Our study, like a previous one, showed that the factors associated with primary dysmenorrhea include a history of heavy menstrual flow and irregular menstrual cycles [11]. However, our study is the first to reveal a strong correlation between PCOM and primary dysmenorrhea. The clinical significance of PCOM remains unclear, especially in young women. Previous studies posited that PCOM is a pre-PCOS state [12] or a prognostic factor in endometrial cancer for patients treated with progestin [13]. Another study described the correlation between PCOM and endometriosis [14]; however, only a few other similar studies have been conducted.

It is thought that the pain of primary dysmenorrhea may be due to increased prostaglandin levels, which causes increased myometrial and uterine blood vessel contractions, thereby producing a relative ischemic uterine state [15]. The 


\section{Obstetrics \& Gynecology Science}

Jee Young Jeong, et al. PCOM and primary dysmenorrhea

pain of primary dysmenorrhea is also known to be associated with sensitization of pain fibers. Peripheral factors and central factors, such as brain-derived neurotrophic factor, were suggested as key factors in the central sensitization of pain in women with primary dysmenorrhea [16]. One possible explanation for the correlation between PCOM and primary dysmenorrhea may be uterine endothelial dysfunction. Increased serum levels of asymmetric dimethylarginine (ADMA), which is established as a strong marker of endothelial dysfunction in atherosclerosis, has been reported in patients with primary dysmenorrhea [17]. Regarding pain, it has been posited that arginine metabolism is 1 of the key mechanisms of pain development in patients with sickle cell disease, a hemoglobinopathy with pain as a disease hallmark. Arginine is thought to play a key role in multiple metabolic processes, particularly with its contribution to endothelial dysfunction and vessel occlusion in sickle cell disease [18]. The same ADMA study group later demonstrated a positive correlation between serum ADMA and serum anti-Mullerian hormone (AMH) in young women with primary dysmenorrhea. They suggested that $A D M A$, like $A M H$, may be used as an ovarian reserve marker [19]. However, it is unclear how serum ADMA and $\mathrm{AMH}$ are connected in the larger context of PCOM, except that they share the same bone morphogenetic protein pathway for signal transduction. The correlation between PCOM and serum AMH is well known, even though the patients in a few of the studies only had PCOM but not PCOS [20].

The relationship between heavy menstruation and pain as an inflammatory response caused by increased prostaglandin synthesis is well established. The serum levels of prostaglandins, specifically prostaglandin E2 [21] or F2a [22], are higher in women with heavy menstrual bleeding (HMB) as shown by data compiled over 30 years. Nonsteroidal antiinflammatory drugs (NSAIDs) help reduce HMB by inhibiting cyclo-oxygenase, thereby reducing prostaglandin production $[21,23]$. Increased prostaglandin level is a common finding in dysmenorrhea and HMB. The strong correlation between heavy menstrual flow and severe dysmenorrhea shown by our study may be explained by increased prostaglandin level.

Our study reports the unique finding that PCOM is strongly associated with the severity of pain in women with primary dysmenorrhea; however, its retrospective nature is a limitation. It is possible that pain was caused by undiagnosed secondary causes such as endometriosis since we did not routinely perform diagnostic laparoscopy. Our study is also limited by the fact that primary dysmenorrhea is a clinical diagnosis made on the basis of menstrual symptoms and by excluding pelvic pathologies that may cause pain.

In conclusion, PCOM and heavy menstrual flow may positively correlate with the severity of pain in young women with primary dysmenorrhea. Since PCOM may play a role in the development of menstrual pain, patients with PCOM should be under active surveillance with resources for prompt pain management readily available. It may also be necessary to further investigate the molecular mechanisms of pain development in primary dysmenorrhea.

\section{Conflict of interest}

No potential conflict of interest relevant to this article was reported.

\section{Ethical approval}

The study was approved by the Institutional Review Board of Severance Hospital, Yonsei University College of Medicine (IRB No. 4-2015-0873) and performed in accordance with the principles of the Declaration of Helsinki. Written informed consents were obtained.

\section{References}

1. lacovides S, Avidon I, Baker FC. What we know about primary dysmenorrhea today: a critical review. Hum Reprod Update 2015;21:762-78.

2. Campbell MA, McGrath PJ. Use of medication by adolescents for the management of menstrual discomfort. Arch Pediatr Adolesc Med 1997;151:905-13.

3. Rotterdam ESHRE/ASRM-Sponsored PCOS consensus workshop group. Revised 2003 consensus on diagnostic criteria and long-term health risks related to polycystic ovary syndrome (PCOS). Hum Reprod 2004;19:41-7.

4. Polson DW, Adams J, Wadsworth J, Franks S. Polycystic ovaries--a common finding in normal women. Lancet 1988;1:870-2.

5. Rosenfield RL. The polycystic ovary morphology-polycystic ovary syndrome spectrum. J Pediatr Adolesc Gynecol 


\section{Obstetrics \& Gynecology Science}

Vol. 62, No. 5, 2019

2015;28:412-9.

6. Youngster M, Ward VL, Blood EA, Barnewolt CE, Emans SJ, Divasta AD. Utility of ultrasound in the diagnosis of polycystic ovary syndrome in adolescents. Fertil Steril 2014;102:1432-8.

7. Codner E, Villarroel C, Eyzaguirre FC, López P, Merino PM, Pérez-Bravo $F$, et al. Polycystic ovarian morphology in postmenarchal adolescents. Fertil Steril 2011;95:702706.e1-2.

8. Dewailly D, Lujan ME, Carmina E, Cedars MI, Laven J, Norman RJ, et al. Definition and significance of polycystic ovarian morphology: a task force report from the Androgen Excess and Polycystic Ovary Syndrome Society. Hum Reprod Update 2014;20:334-52.

9. Clayton RN, Ogden V, Hodgkinson J, Worswick L, Rodin DA, Dyer $S$, et al. How common are polycystic ovaries in normal women and what is their significance for the fertility of the population? Clin Endocrinol (Oxf) 1992;37:127-34.

10. Fritz MA, Speroff L. Clinical gynecologic endocrinology and infertility. 8th ed. Philadelphia (PA): Lippincott Williams \& Wilkins; 2011.

11. Tomás-Rodríguez MI, Palazón-Bru A, Martínez-St John DR, Navarro-Cremades F, Toledo-Marhuenda JV, Gil-Guillén VF. Factors associated with increased pain in primary dysmenorrhea: analysis using a multivariate ordered logistic regression model. J Pediatr Adolesc Gynecol 2017;30:199-202.

12. Tena G, Moran C, Romero R, Moran S. Ovarian morphology and endocrine function in polycystic ovary syndrome. Arch Gynecol Obstet 2011;284:1443-8.

13. Fukui $Y$, Taguchi A, Adachi K, Sato M, Kawata A, Tanikawa $M$, et al. Polycystic ovarian morphology may be a positive prognostic factor in patients with endometrial cancer who achieved complete remission after fertilitysparing therapy with progestin. Asian Pac J Cancer Prev 2017;18:3111-6.

14. Brincat M, Galea R, Buhagiar A. Polycystic ovaries and endometriosis: a possible connection. Br J Obstet Gyn- aecol 1994;101:346-8.

15. Creatsas G, Deligeoroglou E, Zachari A, Loutradis D, Papadimitriou T, Miras K, et al. Prostaglandins: PGF2 alpha, PGE2, 6-keto-PGF1 alpha and TXB2 serum levels in dysmenorrheic adolescents before, during and after treatment with oral contraceptives. Eur J Obstet Gynecol Reprod Biol 1990;36:292-8.

16. Lee LC, Tu CH, Chen LF, Shen HD, Chao HT, Lin MW, et al. Association of brain-derived neurotrophic factor gene Val66Met polymorphism with primary dysmenorrhea. PLoS One 2014;9:e112766.

17. Akdemir N, Cinemre H, Bilir C, Akin O, Akdemir R. Increased serum asymmetric dimethylarginine levels in primary dysmenorrhea. Gynecol Obstet Invest 2010;69:153-6.

18. Bakshi N, Morris CR. The role of the arginine metabolome in pain: implications for sickle cell disease. J Pain Res 2016;9:167-75.

19. Akdemir N, Cinemre FB, Bostancı MS, Cinemre $H$, Ünal $\mathrm{O}$, Ozden $\mathrm{S}$, et al. The correlation of serum asymmetric dimethylarginine and anti-Müllerian hormone in primary dysmenorrhea. Kaohsiung J Med Sci 2016;32:414-9.

20. Villarroel C, Merino PM, López P, Eyzaguirre FC, Van Velzen $A$, Iñiguez $G$, et al. Polycystic ovarian morphology in adolescents with regular menstrual cycles is associated with elevated anti-Mullerian hormone. Hum Reprod 2011;26:2861-8.

21. López Bernal A, Buckley S, Rees CM, Marshall JM. Meclofenamate inhibits prostaglandin $\mathrm{E}$ binding and adenylyl cyclase activation in human myometrium. J Endocrinol 1991;129:439-45.

22. Mäkäräinen L, Ylikorkala O. Primary and myoma-associated menorrhagia: role of prostaglandins and effects of ibuprofen. Br J Obstet Gynaecol 1986;93:974-8.

23. Rees MC, DiMarzo V, Tippins JR, Morris HR, Turnbull AC. Leukotriene release by endometrium and myometrium throughout the menstrual cycle in dysmenorrhoea and menorrhagia. J Endocrinol 1987;113:291-5. 